\title{
FUNCTORIAL CHARACTERIZATIONS OF PONTRYAGIN DUALITY $\left({ }^{1}\right)$
}

\author{
BY \\ DAVID W. ROEDER $\left({ }^{2}\right)$
}

\begin{abstract}
Let $\mathscr{L}$ be the category of locally compact abelian groups, with continuous homomorphisms as morphisms. Let $\chi: \mathscr{L} \rightarrow \mathscr{L}$ denote the contravariant functor which assigns to each object in $\mathscr{L}$ its character group and to each morphism its adjoint morphism. The Pontryagin duality theorem is then the statement that $\chi \circ \chi$ is naturally equivalent to the identity functor in $\mathscr{L}$. We characterize $\chi$ by giving necessary and sufficient conditions for an arbitrary contravariant functor $\varphi: \mathscr{L} \rightarrow \mathscr{L}$ to be naturally equivalent to $\chi$. A sequence of morphisms is called proper exact if it is exact in the algebraic sense and is composed of morphisms each of which is open considered as a function onto its image. A pseudo-natural transformation between two functors in $\mathscr{L}$ differs from a natural transformation in that the connecting maps are not required to be morphisms in $\mathscr{L}$. We study and classify pseudo-natural transformations in $\mathscr{L}$ and use this to prove that ( $R$ denotes the real numbers) $\varphi$ is naturally equivalent to $\chi$ if and only if the following three statements are all true:

(1) $\varphi(R)$ is isomorphic to $R$,

(2) $\varphi$ takes short proper exact sequences to short proper exact sequences, and

(3) $\varphi$ takes inductive limits of discrete groups to projective limits and takes projective limits of compact groups to inductive limits.

From this we prove that $\varphi$ is naturally equivalent to $\chi$ if and only if $\varphi$ is a category equivalence.
\end{abstract}

Pontryagin duality deals with the relationship between a locally compact abelian group $G$ and its character group $\hat{G}$. This correspondence $G \mapsto \hat{G}$ extends to a contravariant functor, which we denote by $\chi$, from the category $\mathscr{L}$ of locally compact abelian groups to itself. In this paper we give three separate characterizations of $\chi$

Presented in part to the Society, January 23,1969 , under the title Two characterizations of Pontryagin duality; received by the editors September 15, 1969 and, in revised form, March 31, 1970.

AMS 1970 subject classifications. Primary 22B05, 43A40, 43A95, 18-XX, 18B99; Secondary 18A20, 18A30, 18E05.

Key words and phrases. Pontryagin duality, locally compact abelian group, character group, compact open topology, additive category, functor, natural transformation, natural equivalence, direct sum, inductive limit, projective limit, proper exact sequence, compactly generated group, group without small subgroups.

(1) Most of the results in this paper are taken from the author's doctoral dissertation, written at the University of California at Santa Barbara under the guidance of Professor Ky Fan. The author wishes to thank Professor Fan for suggesting the problem and for his patience and encouragement throughout. The research presented here was supported in part by NSF grants GP-5578 and GP-8394.

$\left(^{2}\right)$ The author wishes to express his thanks to the referee, some of whose helpful suggestions appear in $\$ 5$.

Copyright (C) 1971, American Mathematical Society 
as a contravariant functor from $\mathscr{L}$ to $\mathscr{L} . \S 1$ presents properties of $\chi$, two systems of axioms chosen from these properties, and pertinent definitions. In $\S 2$ we introduce the definition of pseudo-natural transformation between two functors in $\mathscr{L}$, which is more general than the definition of natural transformation in that the connecting maps need not be morphisms in $\mathscr{L}$. We study and classify pseudo-natural transformations between $\chi$ and arbitrary contravariant functors in $\mathscr{L}$. $\S 3$ and 4 contain the main results; in $\$ 3$ we utilize pseudo-natural transformations in proving that any functor which satisfies Axiom System I or II is naturally equivalent to $\chi$, and in $\S 4$ we prove that any contravariant equivalence in $\mathscr{L}$ is naturally equivalent to $\chi$ by showing that such a functor satisfies Axiom System I. In $\S 5$ some comments and variations on the main results are given $\left({ }^{2}\right)$. In $\$ 6$ we present two theorems which classify pseudo-natural transformations between the identity functor in $\mathscr{L}$ and arbitrary covariant functors in $\mathscr{L}$; these results are analogous to the results in $\S 2$.

1. Definitions and axioms. For the definitions of category, functor and related notions, the reader may consult [1], [5] and [6]. We use the additive notation for all group operations throughout this paper. Let $\mathscr{L}$ denote the category of locally compact abelian topological groups, with morphism meaning continuous homomorphism. The identity morphism on $G$ is denoted by $1_{G}$. The notation $G \simeq H$ means $G$ is topologically isomorphic to $H$; that is, $G$ is isomorphic to $H$ in $\mathscr{L}$. We use the terms injection and surjection for morphisms which are set-theoretically one-to-one and onto, respectively.

We denote the additive group of real numbers with the usual topology by $R$, and the subgroup consisting of the integers with the discrete topology by $Z$. We let $T=R / Z$.

Hom $(G, H)$ for two objects $G$ and $H$ of $\mathscr{L}$ denotes the set of morphisms from $G$ to $H$. For $f$ and $g$ elements of $\operatorname{Hom}(G, H)$ we define the morphism

$$
f+g \in \operatorname{Hom}(G, H)
$$

as follows:

$$
(f+g)(x)=f(x)+g(x) \text { for } x \in G .
$$

With this operation $\operatorname{Hom}(G, H)$ becomes an abelian group, and $\mathscr{L}$ becomes an additive category. Furthermore, providing $\operatorname{Hom}(G, H)$ with the compact-open topology makes it into a Hausdorff (not necessarily locally compact) topological group. We shall consistently assume that $\operatorname{Hom}(G, H)$ is endowed with this particular topological group structure. In the special cases when $G \simeq Z$ or $H \simeq T$, then $\operatorname{Hom}(G, H)$ is a locally compact abelian group and is therefore an object of $\mathscr{L}$. If $\varphi: \mathscr{L} \rightarrow \mathscr{L}$ is any functor, then $\varphi_{G, H}$ denotes either the map

$$
\varphi_{G, H}: \operatorname{Hom}(G, H) \rightarrow \operatorname{Hom}(\varphi(G), \varphi(H))
$$

or

$$
\varphi_{G, H}: \operatorname{Hom}(G, H) \rightarrow \operatorname{Hom}(\varphi(H), \varphi(G))
$$


(depending on whether $\varphi$ is covariant or contravariant) induced by $\varphi$ on $\operatorname{Hom}(G, H)$. The subscripts may be omitted if no confusion arises.

Definition 1. Let $\chi$ be the Pontryagin character group functor in $\mathscr{L}$. That is, for $G$ an object of $\mathscr{L}$, we have

$$
\chi(G)=\hat{G}=\operatorname{Hom}(G, T)
$$

and for $f \in \operatorname{Hom}(G, H)$ we define $\chi_{G, H}(f) \in \operatorname{Hom}(\chi(H), \chi(G))$ by

$$
[\chi(f)](\alpha)=\left[\chi_{G, H}(f)\right](\alpha)=\alpha \circ f \text { for } \alpha \in \chi(H)=\operatorname{Hom}(G, T) .
$$

The fact that $\chi$ is a contravariant functor from $\mathscr{L}$ to $\mathscr{L}$ is easily established. Much literature has been devoted to the remarkable properties of this functor.

Definition 2. Let $\iota$ denote the identity functor in $\mathscr{L}$. For each object $G$ of $\mathscr{L}$, let $\rho_{G}: G \rightarrow \chi(\chi(G))$ be the natural homomorphism defined by

$$
\left[\rho_{G}(x)\right](\alpha)=\alpha(x) \text { for } x \in G, \alpha \in \chi(G) .
$$

Then $\rho=\left\{\rho_{G} ; G\right.$ an object of $\left.\mathscr{L}\right\}$ is a natural transformation from the covariant functor $\iota$ to the covariant functor $\chi^{2}=\chi \circ \chi$. The Pontryagin duality theorem states that $\rho: \iota \rightarrow \chi^{2}$ is in fact a natural equivalence between functors. This duality theorem is, of course, a crucial property of $\chi$.

Let $\varphi: \mathscr{L} \rightarrow \mathscr{L}$ be an arbitrary contravariant functor. Our object is to find sets of additional conditions which, when imposed on $\varphi$, force $\varphi$ to be the "same" functor as $\chi$; categorically speaking, this means that $\varphi$ is to be naturally equivalent to $\chi$. Our next task is to define several concepts which will be of use in characterizing $\chi$.

Definition 3. Let $\varphi: \mathscr{L} \rightarrow \mathscr{L}$ be a functor. We say $\varphi$ is a category equivalence if there exists a functor $\psi: \mathscr{L} \rightarrow \mathscr{L}$ and two natural equivalences $\rho_{1}: \iota \rightarrow \psi \circ \varphi$ and $\rho_{2}: \iota \rightarrow \varphi \circ \psi$. In this case $\psi$ is an inverse of $\varphi$. If $\varphi=\psi$, then we say $\varphi$ is a duality functor. Proposition 10.1 in [6] shows that $\varphi$ is a category equivalence as defined here if and only if the following two conditions are satisfied:

(1) Each function $\varphi_{G, H}$ is bijective for $G, H$ in $\mathscr{L}$.

(2) For every object $G$ of $\mathscr{L}$, there is an object $H$ of $\mathscr{L}$ such that $\varphi(H) \simeq G$.

Definition 4. A morphism $f: G \rightarrow H$ is called proper if $f$ is open regarded as a function from $G$ to its image in $H$. A sequence

$$
\longrightarrow \stackrel{f}{\longrightarrow} B \stackrel{g}{\longrightarrow} C \longrightarrow
$$

of objects and morphisms in $\mathscr{L}$ is said to be proper exact at $B$ if the kernel of $g$ coincides with the image of $f$ and both $f$ and $g$ are proper morphisms. A functor $\varphi$ from $\mathscr{L}$ to $\mathscr{L}$ is called a proper exact functor if for every short proper exact sequence

$$
0 \longrightarrow A \stackrel{f}{\longrightarrow} B \stackrel{g}{\longrightarrow} C \longrightarrow 0
$$


in $\mathscr{L}$, the induced sequence

$$
0 \longrightarrow \varphi(A) \stackrel{\varphi(f)}{\longrightarrow} \varphi(B) \stackrel{\varphi(g)}{\longrightarrow} \varphi(C) \longrightarrow 0
$$

or

$$
0 \longleftarrow \varphi(A) \stackrel{\varphi(f)}{\longleftarrow} \varphi(B) \stackrel{\varphi(g)}{\longleftarrow} \varphi(C) \longleftarrow 0
$$

(depending on whether $\varphi$ is covariant or contravariant) is also a short proper exact sequence. (This definition appears in [7].)

Definition 5. Let $\varphi: \mathscr{L} \rightarrow \mathscr{L}$ be a functor. We say $\varphi$ is topological if $\varphi_{G, H}$ is a continuous function for every pair $G, H$ of objects in $\mathscr{L}$, where $\operatorname{Hom}(G, H)$ is given the compact-open topology introduced before. (Recall that $\varphi$ is additive if each $\varphi_{G, H}$ is a homomorphism between abelian groups.)

Definition 6. Let $G$ and $H$ be objects in $\mathscr{L}$ and let $G \oplus H$ be the ordinary "direct sum" of $G$ and $H$ (i.e., the product group with the product topology), let $i_{G}$ and $i_{H}$ be the canonical embeddings of $G$ and $H$, respectively, in $G \oplus H$ and let $p_{G}$ and $p_{H}$ be the canonical projections of $G \oplus H$ onto $G$ and $H$, respectively. $G \oplus H$ can be characterized in $\mathscr{L}$ by the "direct sum diagram"

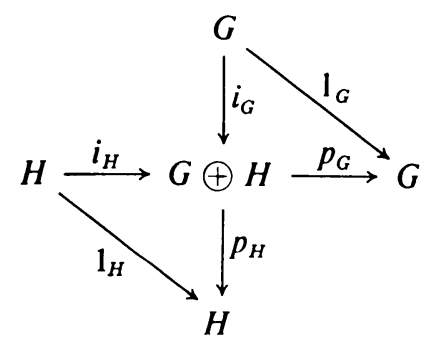

together with the requirements that the diagram be commutative and the morphisms satisfy the equation

$$
i_{G} \circ p_{G}+i_{H} \circ p_{H}=1_{G \oplus H}
$$

(see [5, p. 250]).

The direct sum is unique up to canonical isomorphism in $\mathscr{L}$. Now if $g: G \rightarrow L$ and $h: H \rightarrow L$ are morphisms in $\mathscr{L}$, then we define the morphism

$$
(g, h) \in \operatorname{Hom}(G \oplus H, L)
$$

by $(g, h)=g \circ p_{G}+h \circ p_{H}$. Then we have $(g, h) \circ i_{G}=g$ and $(g, h) \circ i_{H}=h$. Moreover, $(g, h)$ is uniquely determined by these conditions because if $f: G \oplus H \rightarrow L$ is any morphism such that $f \circ i_{G}=g$ and $f \circ i_{H}=h$, then we have

$$
f=f \circ\left(i_{G} \circ p_{G}+i_{H} \circ p_{H}\right)=g \circ p_{G}+h \circ p_{H}=(g, h) .
$$

This merely shows that $\left(G \oplus H, i_{G}, i_{H}\right)$ is a categorical sum of $G$ and $H$. Likewise, 
it can be seen that $\left(G \oplus H, p_{G}, p_{H}\right)$ is a categorical product of $G$ and $H$; that is, if $g: K \rightarrow G$ and $h: K \rightarrow H$ are morphisms, then

$$
\left(\begin{array}{l}
g \\
h
\end{array}\right)=i_{G} \circ g+i_{H} \circ h: K \rightarrow G \oplus H
$$

is the only morphism such that $p_{G} \circ\left(\begin{array}{l}g \\ h\end{array}\right)=g$ and $p_{H} \circ\left(\begin{array}{l}g \\ h\end{array}\right)=h$.

Definition 7. For the purposes of this paper, inductive limit in $\mathscr{L}$ will mean the usual categorical limit (if it exists) of a direct system in $\mathscr{L}$, which consists of a set $\left\{G_{\alpha}\right\}$ of objects of $\mathscr{L}$ indexed by a directed set $A$ together with a set of morphisms $\left\{f_{\alpha \beta}\right\}$ such that $f_{\alpha \beta} \in \operatorname{Hom}\left(G_{\alpha}, G_{\beta}\right)$ whenever $\alpha \leqq \beta$ and $f_{\beta \gamma} \circ f_{\alpha \beta}=f_{\alpha \gamma}$ whenever $\alpha \leqq \beta \leqq \gamma$. A proper inductive limit in $\mathscr{L}$ is the inductive limit (which automatically will exist) of a proper direct system (each $f_{\alpha \beta}$ is a proper injection whose image is an open subgroup of $G_{\beta}$ ). Dually, the terms projective limit and inverse system are obtained by reversing the directions of the arrows and order of composition of morphisms in the above. The term proper projective limit is reserved for a projective limit of an inverse system $\left(\left\{G_{\alpha}\right\},\left\{f_{\alpha \beta}\right\}\right)$ in which each $f_{\alpha \beta}: G_{\beta} \rightarrow G_{\alpha}$ is a proper surjection with compact kernel, called a proper inverse system.

Our first proposition is merely a summary of important functorial properties of $\chi$ which will be useful in our later results. To avoid possible confusion, we note that to say "the functor $\varphi$ preserves direct sums" means that $\varphi$ takes the direct sum diagram and equation for $G \oplus H$ to the direct sum diagram and equation for $\varphi(G) \oplus \varphi(H)$. Similar interpretations are to be applied to other such statements about functors.

Proposition 1. The following are true concerning the functor $\chi$ :

(1) $\chi$ is a duality functor.

(2) $\chi$ is a contravariant category equivalence.

(3) $\chi$ is an additive functor.

(4) $\chi$ is a topological functor.

(5) $\chi(Z) \simeq T, \chi(T) \simeq Z, \chi(R) \simeq R$.

(6) $\chi$ is a proper exact functor.

(7) $\chi$ preserves direct sums.

(8) $\chi$ takes inductive limits to projective limits and projective limits to inductive limits.

Proof. (1), (2), (5) and (7) are well known. (3), (6) and a weaker version of (8) for proper inductive limits and proper projective limits have been demonstrated before (see [10]). In fact, Theorem 5 will show that (2) alone implies all the others. Regarding (4), Suzuki [9] mentions that each $\chi_{G, H}$ is actually a topological isomorphism. However, we present a proof of (4) here, since we have not seen it in the literature.

Let $G$ and $H$ be objects in $\mathscr{L}$. We know a base for the neighborhoods of the identity in $\operatorname{Hom}(G, H)$ is given by the set of all $N(K, V)$, where $K$ runs through the compact subsets of $G$ and $V$ runs through a base of neighborhoods of the identity 
in $H$, and where $N(K, V)$ is the set of all $f \in \operatorname{Hom}(G, H)$ for which $f(K)$ is contained in $V$. Accordingly, any neighborhood of the identity in Hom $(\chi(H), \chi(G))$ contains a neighborhood of the form

$$
N(F, N(K, U))
$$

where $F$ is a compact set in $\chi(H), K$ is a compact set in $G$, and $U$ is a neighborhood of the identity in $T$. Now, by well-known properties of compact-open topologies for locally compact spaces, the map $\gamma: H \times \chi(H) \rightarrow T$ given by $(x, \alpha) \mapsto \alpha(x)$ is continuous. Under this map we have

$$
\gamma(\{0\} \times F)=\{0\} \subset U \subset T ;
$$

therefore there is a neighborhood $W$ of the identity in $H$ such that $\gamma(W \times F) \subset U$. Now, let $f$ be an element of $N(K, W)$, a neighborhood of the identity in $\operatorname{Hom}(G, H)$. Let $x \in K$ and $\alpha \in F$; then

$$
[\chi(f)](\alpha)=\alpha \circ f, \quad \text { and } \quad(\alpha \circ f)(x) \in \alpha(W)=\gamma(W \times\{\alpha\}) \subset \gamma(W \times F) \subset U .
$$

Thus $\chi(f) \in N(F, N(K, U))$. Q.E.D.

Proposition 2. Let $\varphi: \mathscr{L} \rightarrow \mathscr{L}$ be a contravariant functor and suppose there is a natural equivalence $\tau: \chi \rightarrow \varphi$. Then statements (1) through (8) of Proposition 1 also hold for $\varphi$.

Proof. Let $G$ and $H$ be objects of $\mathscr{L}$ and consider the following diagram where $f \in \operatorname{Hom}(G, H)$ and $\iota$ and $\rho$ are defined in Definition 2:

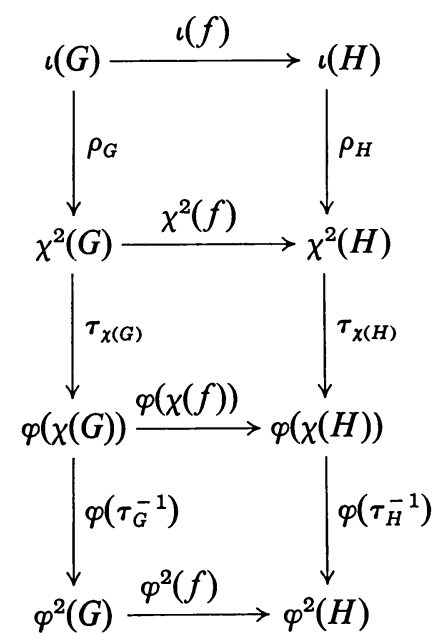

The morphisms represented by vertical arrows are all isomorphisms in $\mathscr{L}$ by hypothesis. The upper and middle small rectangles commute because $\rho$ and $\tau$ are natural transformations, and the lower small rectangle commutes because it comes 
from applying $\varphi$ to the following commutative diagram:

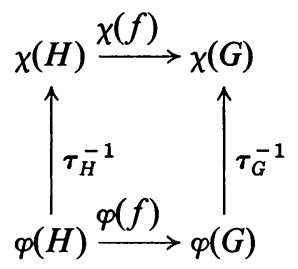

Thus the large outside rectangle of the first diagram is commutative. This means that the collection of all morphisms of the form

$$
\varphi\left(\tau_{G}^{-1}\right) \circ \tau_{\chi(G)} \circ \rho_{G}: \iota(G) \rightarrow \varphi^{2}(G)
$$

is a natural equivalence between $\iota$ and $\varphi^{2}$. This shows that statement (1) holds for $\varphi$.

Now, statement (2) follows directly from (1). Statement (5) is obvious. Statements (3), (6), (7) and (8) follow from standard arguments developed in category theory, analogous to the proof of statement (1). Statement (4) is different in that it does not concern a familiar categorical notion. We prove (4) by examining the diagram

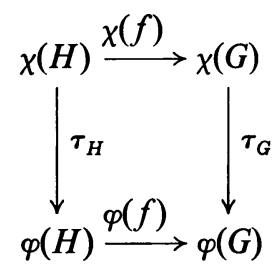

for $f \in \operatorname{Hom}(G, H)$. This shows that $\varphi(f)=\tau_{G} \circ \chi(f) \circ \tau_{H}^{-1}$. Thus we have a bijective map

$$
\text { Hom }(\chi(H), \chi(G)) \rightarrow \operatorname{Hom}(\varphi(H), \varphi(G))
$$

given by the correspondence

$$
\chi(f) \mapsto \tau_{G} \circ \chi(f) \circ \tau_{H}^{-1} .
$$

This map is obviously a group isomorphism and is also a homeomorphism, since an open set in $\operatorname{Hom}(\chi(H), \chi(G))$ of the form $N(K, V)$ is taken onto the open set $N\left(\tau_{H}(K), \tau_{G}(V)\right)$, and vice versa. Thus the map $\varphi_{G, H}: f \mapsto \varphi(f)$ is a composition of the two maps $f \mapsto \chi(f)$ and $\chi(f) \mapsto \varphi(f)$, both of which are topological isomorphisms. Q.E.D.

Let $\varphi: \mathscr{L} \rightarrow \mathscr{L}$ be a contravariant functor. We present two systems of axioms for $\varphi$.

Axiom System I (three axioms).

Ia. $\varphi(R) \simeq R$.

Ib. $\varphi$ is a proper exact functor.

Ic. $\varphi$ takes proper inductive limits of discrete groups to projective limits and takes proper projective limits of compact groups to inductive limits. 
Axiom System II (four axioms).

IIa. $\varphi(R) \simeq R$.

IIb. $\varphi$ takes the proper exact sequences

$$
0 \longrightarrow Z \stackrel{i}{\longrightarrow} R \stackrel{p}{\longrightarrow} T \longrightarrow 0
$$

and

$$
0 \longrightarrow Z \stackrel{n \cdot 1_{Z}}{\longrightarrow} Z \stackrel{p_{n}}{\longrightarrow} F_{n} \longrightarrow 0,
$$

where $F_{n}$ is a cyclic group of order $n$, to short proper exact sequences.

IIc. $\varphi$ takes proper inductive limits of discrete groups to projective limits and takes all proper projective limits to inductive limits.

IId. $\varphi$ is an additive functor.

Our task is to demonstrate that any contravariant functor in $\mathscr{L}$ which either satisfies one of these axiom systems or is a category equivalence is naturally equivalent to $\chi$. Proposition 2 demonstrates the necessity of each axiom.

\section{Construction of pseudo-natural transformations.}

Definition 8. Let $\varphi$ and $\psi$ be two contravariant functors from $\mathscr{L}$ to $\mathscr{L}$. Suppose we have a collection $\sigma=\left\{\sigma_{G}: G\right.$ an object in $\left.\mathscr{L}\right\}$ such that each $\sigma_{G}$ is a function (but not necessarily a morphism in $\mathscr{L}$ ) from $\varphi(G)$ to $\psi(G)$. Then we call $\sigma$ a pseudonatural transformation from $\varphi$ to $\psi$, denoted $\sigma: \varphi \rightarrow \psi$, if for each pair $G, H$ of objects of $\mathscr{L}$ and every $f \in \operatorname{Hom}(G, H)$, we have

$$
\psi(f) \circ \sigma_{H}=\sigma_{G} \circ \varphi(f) ;
$$

that is, the following diagram is commutative:

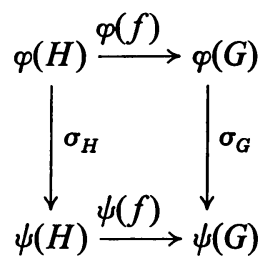

In a similar manner we define the notion of pseudo-natural transformation from one covariant functor to another.

We remark that if $\mathscr{E}$ is the category of sets and $F: \mathscr{L} \rightarrow \mathscr{E}$ is the "forgetful" functor which assigns to each object of $\mathscr{L}$ its underlying set, then the study of pseudo-natural transformations between $\varphi$ and $\psi$ is equivalent to the study of natural transformations between the functors $F \circ \varphi$ and $F \circ \psi$. In this sense, all but the last statement of Theorem 1 below is a special case of Theorem 1.6 in [1].

For any object $G$ of $\mathscr{L}$, let $\xi_{G}$ : $\operatorname{Hom}(Z, G) \rightarrow G$ be the natural topological isomorphism given by $\xi_{G}(\alpha)=\alpha(1)$ for $\alpha \in \operatorname{Hom}(Z, G)$. (We use the symbol 1 for 
the integer $1 \in Z$ and the symbol $1_{G}$ for the identity map on $G$, where $G$ is an object in $\mathscr{L}$.)

We point out at this time that $1_{T}$, the identity morphism on $T$, is regarded as an element of $\operatorname{Hom}(T, T)=\chi(T)$.

THEOREM 1. Let $\varphi: \mathscr{L} \rightarrow \mathscr{L}$ be a contravariant functor. Let $s$ be any element of $\varphi(T)$. Then there exists exactly one pseudo-natural transformation $\tau^{s}: \chi \rightarrow \varphi$ satisfying the equation $\tau_{T}^{s}\left(1_{T}\right)=s$. Each $\tau_{G}^{s}: \chi(G) \rightarrow \varphi(G)$ is defined by the formula

$$
\tau_{G}^{s}(\alpha)=\left[\varphi_{G, T}(\alpha)\right](s) \text { for } \alpha \in \chi(G)=\operatorname{Hom}(G, T) .
$$

Thus there is a one-to-one correspondence between pseudo-natural transformations $\tau^{s}: \chi \rightarrow \varphi$ and elements $s$ of $\varphi(T)$. If $\varphi$ is a topological additive functor, then each $\tau^{s}$ is in fact a natural transformation.

Proof. Let $G$ and $H$ be objects in $\mathscr{L}$, let $f \in \operatorname{Hom}(G, H)$ and let $\alpha \in \chi(H)$. Let $\tau_{G}^{s}$ and $\tau_{H}^{s}$ be defined as above. Then

$$
\begin{aligned}
{\left[\varphi(f) \circ \tau_{H}^{s}\right](\alpha) } & =\left[\varphi(f) \circ\left(\varphi_{H, T}(\alpha)\right)\right](s) \\
& =\left[\varphi_{G, T}(\alpha \circ f)\right](s)=\tau_{G}^{s}(\alpha \circ f) \\
& =\left[\tau_{G}^{s} \circ(\chi(f))\right](\alpha) .
\end{aligned}
$$

Thus $\tau^{s}$ as defined in the theorem is a pseudo-natural transformation from $\chi$ to $\varphi$. Furthermore,

$$
\tau_{T}^{s}\left(1_{T}\right)=\left[\varphi_{T, T}\left(1_{T}\right)\right](s)=1_{\varphi(T)}(s)=s .
$$

Now let $\tau: \chi \rightarrow \varphi$ be any pseudo-natural transformation and let $\tau_{T}\left(1_{T}\right)=s$. Let $\alpha \in \chi(G)$, where $G$ is any object in $\mathscr{L}$. Since $\tau$ is a pseudo-natural transformation, the following diagram commutes for the morphism $\alpha \in \operatorname{Hom}(G, T)$ :

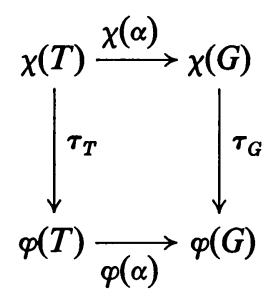

Therefore we have

$$
\begin{aligned}
\tau_{G}^{s}(\alpha) & =\left[\varphi_{G, T}(\alpha)\right]\left[\tau_{T}\left(1_{T}\right)\right] \\
& =\tau_{G}\left([\chi(\alpha)]\left(1_{T}\right)\right)=\tau_{G}\left(1_{T} \circ \alpha\right)=\tau_{G}(\alpha) .
\end{aligned}
$$

Hence $\tau=\tau^{s}$.

For the last statement of the theorem, we observe that the map $\tau_{G}^{s}$ from $\chi(G)$ to $\varphi(G)$ can be obtained by applying the map $\varphi_{G, T}$ from $\chi(G)=\operatorname{Hom}(G, T)$ to Hom $(\varphi(T), \varphi(G))$ and then applying the map from $\operatorname{Hom}(\varphi(T), \varphi(G))$ to $\varphi(G)$ given by $f \mapsto f(s)$. Q.E.D. 
Corollary 1. Let $\varphi: \mathscr{L} \rightarrow \mathscr{L}$ be a contravariant functor such that $\varphi(T) \simeq Z$ and for every object $G$ of $\mathscr{L}$, the map $\varphi_{G, T}$ is a topological isomorphism. Then $\varphi$ is naturally equivalent to $\chi$.

Proof. Let $s$ be a generator of $\varphi(T)$, and consider the pseudo-natural transformation $\tau^{s}: \chi \rightarrow \varphi$. We observed at the end of the proof to Theorem 1 that each map $\tau_{G}^{s}$ is the composition of two other maps, and in this case both those other maps are topological isomorphisms. Q.E.D.

The preceding theorem shows that the construction and classification of pseudonatural transformations from $\chi$ to a contravariant functor $\varphi$ in $\mathscr{L}$ is quite simple. Unfortunately, constructing pseudo-natural transformations from $\varphi$ to $\chi$ is not so easy. However, we are able to classify such pseudo-natural transformations in terms of what they do to $\varphi(Z)$, as the following result shows.

THEOREM 2. Let $\varphi: \mathscr{L} \rightarrow \mathscr{L}$ be a contravariant functor. Let $\mu$ be a function from $\varphi(Z)$ to $\chi(Z)$. Then the following two conditions are equivalent:

(1) There exists a pseudo-natural transformation $\sigma^{\mu}: \varphi \rightarrow \chi$ such that $\sigma_{Z}^{\mu}=\mu$.

(2) For every object $G$ of $\mathscr{L}$ and every $x \in \varphi(G)$ the following function $G \rightarrow T$ is a morphism in $\mathscr{L}$ :

$$
y \mapsto\left[\xi_{T} \circ \mu \circ\left(\varphi_{Z, G}\left[\xi_{G}^{-1}(y)\right]\right)\right](x) .
$$

Moreover, there is at most one pseudo-natural transformation satisfying condition (1). In fact, if $\sigma^{\mu}$ satisfies (1), then for $G$ in $\mathscr{L}$ and $x \in \varphi(G), \sigma_{G}^{\mu}(x)$ is that element of $\chi(G)$ given by

$$
\left[\sigma_{G}^{\mu}(x)\right](y)=\left[\xi_{T} \circ \mu \circ\left(\varphi_{Z, G}\left[\xi_{G}^{-1}(y)\right]\right)\right](x) \text { for every } y \in G .
$$

In particular, if $\varphi$ is topological and additive and $\mu$ is a morphism in $\mathscr{L}$, then condition (2) is satisfied and the $\sigma^{\mu}$ satisfying condition (1) is actually a natural transformation.

Proof. First, suppose condition (2) holds. Then we define $\sigma_{G}^{\mu}: \varphi(G) \rightarrow \chi(G)$ by condition (3). Let $G$ and $H$ be objects in $\mathscr{L}$, let $x \in \varphi(H)$, let $y \in G$, and let $f \in$ Hom $(G, H)$. Then

$$
\begin{aligned}
{\left[\left(\sigma_{G}^{\mu} \circ \varphi(f)\right)(x)\right](y) } & =\left(\xi_{T} \circ \mu\right)\left(\varphi_{Z, G}\left[\xi_{G}^{-1}(y)\right] \circ \varphi_{G, H}(f)\right)(x) \\
& =\left(\xi_{T} \circ \mu\right)\left[\varphi_{Z, H}\left(f \circ \xi_{G}^{-1}(y)\right)(x)\right],
\end{aligned}
$$

while

$$
\begin{aligned}
{\left[\left(\chi(f) \circ \sigma_{H}^{\mu}\right)(x)\right](y) } & =\left[\sigma_{H}^{\mu}(x) \circ f\right](y)=\left[\sigma_{H}^{\mu}(x)\right](f(y)) \\
& =\left(\xi_{T} \circ \mu\right)\left(\varphi_{z, H}\left[\xi_{H}^{-1}(f(y))\right](x)\right) .
\end{aligned}
$$

We now examine $f \circ \xi_{G}^{-1}(y)$ and $\xi_{H}^{-1}(f(y))$. These are both elements of $\operatorname{Hom}(Z, H)$, so they will be equal if they agree at $1 \in Z$. We see that

$$
\left[f \circ \xi_{G}^{-1}(y)\right](1)=f(y)=\left[\xi_{H}^{-1}(f(y))\right](1) .
$$


Thus $\sigma_{G}^{\mu} \circ \varphi(f)=\chi(f) \circ \sigma_{H}^{\mu}$, so $\sigma^{\mu}$ is a pseudo-natural transformation. In addition, if we let $x \in \varphi(Z)$, we have

$$
\begin{aligned}
{\left[\sigma_{Z}^{\mu}(x)\right](1) } & =\left[\xi_{T} \circ \mu \circ\left(\varphi_{Z, Z}\left[\xi_{\bar{z}}^{-1}(1)\right]\right)\right](x) \\
& =\left(\xi_{T} \circ \mu \circ 1_{\varphi(Z)}\right)(x)=[\mu(x)](1) .
\end{aligned}
$$

Since $\sigma_{Z}^{\mu}(x)$ and $\mu(x)$ are elements of $\chi(Z)$ which agree at 1 , they must be equal. This shows that $\sigma^{\mu}$ satisfies condition (1).

Now let $\sigma: \varphi \rightarrow \chi$ be any pseudo-natural transformation which satisfies (1). Let $G$ be an object in $\mathscr{L}$, let $x \in \varphi(G)$ and let $y \in G$. Since $\sigma$ is a pseudo-natural transformation, the following equation is satisfied by the morphism $\xi_{G}^{-1}(y) \in \operatorname{Hom}(Z, G)$ :

Hence

$$
\sigma_{Z} \circ \varphi_{Z, G}\left[\xi_{G}^{-1}(y)\right]=\chi_{z, G}\left[\xi_{G}^{-1}(y)\right] \circ \sigma_{G} .
$$

$$
\begin{aligned}
{\left[\xi_{T} \circ \mu \circ\left(\varphi_{Z, G}\left[\xi_{G}^{-1}(y)\right]\right)\right](x) } & =\left(\xi_{T} \circ \sigma_{Z} \circ \varphi_{Z, G}\left[\xi_{G}^{-1}(y)\right]\right)(x) \\
& =\left[\left(\chi_{z, G}\left[\xi_{G}^{-1}(y)\right] \circ \sigma_{G}\right)(x)\right](1) \\
& =\left(\sigma_{G}(x) \circ\left[\xi_{G}^{-1}(y)\right]\right)(1)=\left[\sigma_{G}(x)\right](y) .
\end{aligned}
$$

This means that the map $G \rightarrow T$ given in condition (2) is simply the map $y \mapsto$ $\left[\sigma_{G}(x)\right](y)$, so condition (2) is satisfied. Then if we define $\sigma^{\mu}$ according to condition (3), we have just shown that $\sigma^{\mu}=\sigma$.

Now suppose $\varphi$ is topological and additive and $\mu: \varphi(Z) \rightarrow \chi(Z)$ is a morphism. Then clearly condition (2) is satisfied, and so there exists $\sigma^{\mu}: \varphi \rightarrow \chi$ satisfying condition (1). We show an alternate definition of $\sigma^{\mu}$. Let $G$ be an object in $\mathscr{L}$. We define a function $\nu$ from $\operatorname{Hom}(\varphi(G), \varphi(Z))$ to $\operatorname{Hom}(\varphi(G), T)$ by $\nu(f)=\xi_{T} \circ \mu \circ f$. If $N(K, V)$ is a neighborhood of 0 in Hom $(\varphi(G), T)$, then let $U$ be a neighborhood of 0 in $\varphi(Z)$ such that $\left(\xi_{T} \circ \mu\right)(U) \subset V$. Then $f \in N(K, U)$ implies that $\nu(f)$ is an element of $N(K, V)$. Thus $\nu$ is a continuous homomorphism. Let $\lambda=\nu \circ \varphi_{Z, G} \circ \xi_{G}^{-1}$. Then $\lambda$ is a morphism $G \rightarrow \chi \varphi(G)$. Finally set $\delta=\chi(\lambda) \circ \rho_{\varphi(G)} ; \delta$ is a morphism from $\varphi(G)$ to $\chi(G)$. We show that $\delta=\sigma_{G}^{\mu}$. Let $x \in \varphi(G)$ and $y \in G$. Then

$$
\begin{aligned}
{[\delta(x)](y) } & =\left[\left(\chi(\lambda) \circ \rho_{\varphi(G)}\right)(x)\right](y) \\
& =\left[\rho_{\varphi(G)}(x) \circ \lambda\right](y) \\
& =\left[\left(\nu \circ \varphi_{Z, G} \circ \xi_{G}^{-1}\right)(y)\right](x) \\
& =\left[\xi_{T} \circ \mu \circ\left(\varphi_{Z, G}\left[\xi_{G}^{-1}(y)\right]\right)\right](x)=\left[\sigma_{G}^{\mu}(x)\right](y) .
\end{aligned}
$$

Hence $\sigma^{\mu}$ is a natural transformation. Q.E.D.

CoRollary 2. Let $\varphi: \mathscr{L} \rightarrow \mathscr{L}$ be a contravariant functor such that $\varphi(Z) \simeq \chi(Z)$ and for every $G$ in $\mathscr{L}, \varphi_{Z, G}$ is a topological isomorphism. Then $\varphi$ is naturally equivalent to $\chi$.

Proof. By examining the last part of the proof of Theorem 2, one sees that the $\operatorname{map} \lambda: G \rightarrow \chi \varphi(G)$ is an isomorphism in $\mathscr{L}$. Hence $\sigma^{\mu}$ is a natural equivalence. Q.E.D. 
3. Axiom Systems I and II. A word here about the known structure of objects of $\mathscr{L}$ will be useful. By the structure theorem for compactly generated objects of $\mathscr{L}\left[8\right.$, p. 269], such a group is isomorphic with one of the form $Z^{m} \oplus R^{n} \oplus C$, where $m$ and $n$ are nonnegative integers and $C$ is a compact abelian group. Therefore the character group of a compactly generated object of $\mathscr{L}$ is isomorphic with a group of the form $T^{m} \oplus R^{n} \oplus D$, where $D$ is a discrete abelian group. A group of this form is called a group without small subgroups (see [7]). We know that any object of $\mathscr{L}$ is a proper inductive limit of its compactly generated open subgroups. In particular, then, any discrete group is the inductive limit of its finitely generated subgroups. As consequences of these facts and the Pontryagin duality theorem, any object of $\mathscr{L}$ is a proper projective limit of groups without small subgroups, and any compact group in $\mathscr{L}$ is a proper projective limit of groups of the form $T^{m} \oplus F$, where $F$ is a finite group.

Lemma 1. Let $\varphi: \mathscr{L} \rightarrow \mathscr{L}$ be a proper exact functor. Then $\varphi$ preserves direct sums.

Proof. Assume $\varphi$ is contravariant. Notice that in the direct sum diagram for $G \oplus H$ (see Definition 6), the row and column become short proper exact sequences when zeros are added at the beginning and end of each. Applying $\varphi$ to this diagram, we obtain the commutative diagram with exact row and column:

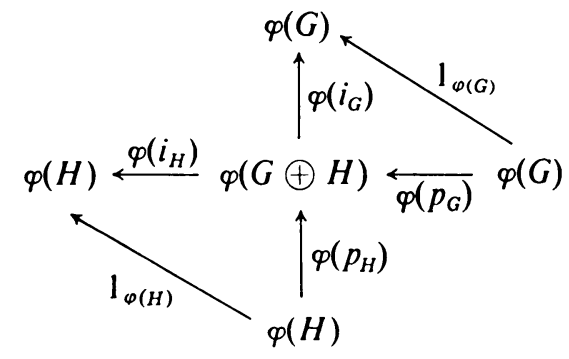

Then, since $\varphi\left(i_{G}\right) \circ \varphi\left(p_{G}\right)=1_{\varphi(G)}$ we may conclude from (6.22) in [2] that $\varphi(G \oplus H)$ is the direct sum of the image of $\varphi\left(p_{G}\right)$ and the kernel of $\varphi\left(i_{G}\right)$; by the exactness this means that $\varphi(G \oplus H)$ is the direct sum of the images of $\varphi\left(p_{G}\right)$ and $\varphi\left(p_{H}\right)$; thus we have the direct sum diagram and equation for $\varphi(G) \oplus \varphi(H)$. Q.E.D.

Lemma 2. Let $\varphi: \mathscr{L} \rightarrow \mathscr{L}$ be a contravariant functor. Then the following are equivalent:

(1) $\varphi$ is additive.

(2) $\varphi$ preserves direct sums.

(3) $\varphi$ takes finite products to sums.

(4) $\varphi$ takes finite sums to products.

Proof. Suppose (1) holds. Then $\varphi$ takes the direct sum diagram and equation for $G \oplus H$ to a direct sum diagram for $\varphi(G) \oplus \varphi(H)$ in which the analogous equation is still true, so $\varphi$ preserves direct sums. 
Suppose (2) holds. The remarks on sums and products following Definition 6 show that (3) and (4) are true.

By the uniqueness, up to isomorphism, of sums and products in any category, we know that any sum or product in $\mathscr{L}$ is automatically a direct sum. Thus if either (3) or (4) is true, then $\varphi$ will take any direct sum into a direct sum, so that (2) will hold.

If (3) and (4) hold, then it is trivially verified that

$$
\varphi(f, g)=\left(\begin{array}{l}
\varphi(f) \\
\varphi(g)
\end{array}\right) \text { and } \varphi\left(\begin{array}{l}
f \\
g
\end{array}\right)=(\varphi(f), \varphi(g))
$$

for any two morphisms $f$ and $g$ with the same range, or same domain, respectively. Also, for $f$ and $g$ elements of $\operatorname{Hom}(G, H)$, an easy computation shows that the morphism $f+g$ can be expressed as either of the following compositions:

$$
\begin{aligned}
& G \stackrel{\left(\begin{array}{l}
1_{G} \\
1_{G}^{G}
\end{array}\right)}{\longrightarrow} G \oplus G \stackrel{(f, g)}{\longrightarrow} H \\
& G \stackrel{\left({ }_{g}^{f}\right)}{\longrightarrow} H \oplus H \stackrel{\left(1_{H}, 1_{H}\right)}{\longrightarrow} H .
\end{aligned}
$$

(This fact appears in [4].) Thus

$$
\begin{aligned}
\varphi(f+g) & =\varphi\left[(f, g) \circ\left(\begin{array}{l}
1_{G} \\
1_{G}
\end{array}\right)\right] \\
& =\left(1_{\varphi(G)}, 1_{\varphi(G)}\right) \circ\left(\begin{array}{l}
\varphi(f) \\
\varphi(g)
\end{array}\right)=\varphi(f)+\varphi(g) . \quad \text { Q.E.D. }
\end{aligned}
$$

THEOREM 3. Any contravariant functor $\varphi: \mathscr{L} \rightarrow \mathscr{L}$ which satisfies Axiom System I is naturally equivalent to the functor $\chi$.

Proof. We first examine the short proper exact sequence

$$
0 \longrightarrow Z \stackrel{i}{\longrightarrow} R \stackrel{p}{\longrightarrow} T \longrightarrow 0
$$

which by hypothesis is taken by $\varphi$ to a short proper exact sequence

$$
0 \longrightarrow \varphi(T) \stackrel{\varphi(p)}{\longrightarrow} R \stackrel{\varphi(i)}{\longrightarrow} \varphi(Z) \longrightarrow 0 .
$$

Thus $\varphi(T)$ is isomorphic to a subgroup of $R$. Suppose $\varphi(T) \simeq R$. Then let $\left\{G_{\alpha}\right\}$ be any infinite collection of objects of $\mathscr{L}$ each of which is isomorphic to $T$, and let $G=\prod_{\alpha} G_{\alpha}$ (with the product topology), a compact group in $\mathscr{L}$. For any finite set $A$ of $\alpha$ 's, let $G_{A}=\prod_{\alpha \in A} G_{\alpha}$ and let $K_{A}=\prod_{\alpha \notin A} G_{\alpha}$. Then $G \simeq G_{A} \oplus K_{A}$ and so $\varphi(G) \simeq$ $\varphi\left(G_{A}\right) \oplus \varphi\left(K_{A}\right)$. By Lemma 1 and Axiom System I we obtain

$$
\varphi\left(G_{A}\right) \simeq \bigoplus_{\alpha \in A} \varphi\left(G_{A}\right) \simeq R^{n}
$$

where $n$ is the cardinality of $A$. Thus the connected component of $\varphi(G)$ contains a subgroup isomorphic to $R^{n}$ for arbitrary positive integers $n$. This contradicts the 
structure theorem for compactly generated objects in $\mathscr{L}$, since a connected group in $\mathscr{L}$ is compactly generated. Therefore $\varphi(T) \simeq R$ is impossible.

Similarly, if $\varphi(Z) \simeq R$, we let $\left\{H_{\alpha}\right\}$ be an infinite collection of groups each isomorphic with $Z$. We let $H=\oplus_{\alpha} H_{\alpha}$ (the algebraic direct sum with the discrete topology). Then applying the above argument we arrive at the fact that $\varphi(H)$ contains a subgroup isomorphic to $R^{n}$, where $n$ can be made arbitrarily large, a contradiction again.

We now know that $\varphi(T)$ is isomorphic to a subgroup of $R$ and $\varphi(Z)$ is isomorphic to a quotient group of $R$ but neither is isomorphic to $R$. The exactness of the sequence

$$
0 \rightarrow \varphi(T) \rightarrow R \rightarrow \varphi(Z) \rightarrow 0
$$

assures us that $\varphi(T) \simeq Z$ and $\varphi(Z) \simeq T$.

Now let $s$ be a generator of $\varphi(T)$ and consider the pseudo-natural transformation $\tau^{s}: \chi \rightarrow \varphi$ defined in Theorem 1. By Lemma $2, \varphi$ is an additive functor. Looking at the definition of $\tau^{s}$ in Theorem 1 , we see that $\tau_{G}^{s}$ is therefore a group homomorphism at least, if not a morphism in $\mathscr{L}$, for each object $G$. We obtain the following commutative diagram with proper exact rows:

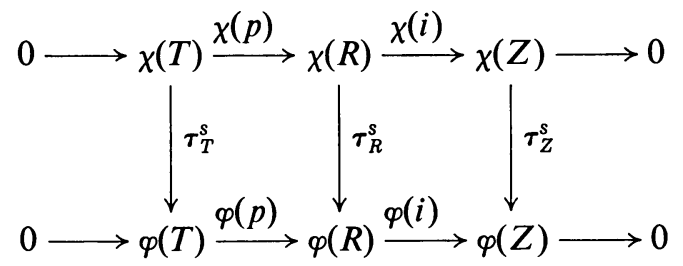

We know that $\tau_{T}^{s}\left(1_{T}\right)=s$, so $\tau_{T}^{s}$ takes a generator of $\chi(T)$ to a generator of $\varphi(T)$. Therefore $\tau_{T}^{s}$ is a topological isomorphism of infinite cyclic groups. Then by the exactness, we know that $\tau_{R}^{s}$ can not be the zero homomorphism. If we can show that $\tau_{R}^{s}$ is a morphism in $\mathscr{L}$, then we will know that $\tau_{R}^{s}$ is an isomorphism in $\mathscr{L}$, since any morphism $R \rightarrow R$ in $\mathscr{L}$ is automatically linear. By the definition of $\tau^{s}$ in Theorem 1 it suffices to show that $\varphi_{R, T}$ is continuous. To do this, we first examine the map $\varphi_{R, R}$. We know that as topological groups $R \simeq \operatorname{Hom}(R, R)$ by the correspondence $t \mapsto m(t)$ where $m(t)$ is multiplication by $t$. Let $f: R \rightarrow \varphi(R)$ be any isomorphism and define the map $f_{0}: \operatorname{Hom}(\varphi(R), \varphi(R)) \rightarrow \operatorname{Hom}(R, R)$ by $f_{0}(\alpha)=$ $f^{-1} \circ \alpha \circ f$. Now, let $\varphi_{0}: R \rightarrow R$ by $\varphi_{0}=m^{-1} \circ f_{0} \circ \varphi_{R, R} \circ m . \varphi_{0}$ is a homomorphism of the additive group structure of $R$, and it is clear that $\varphi_{0}(1)=1$. Using the fact that $m(s t)=m(s) \circ m(t)$ and hence $m^{-1}(\alpha \circ \beta)=m^{-1}(\alpha) \cdot m^{-1}(\beta)$, we see that

$$
\begin{aligned}
\varphi_{0}(s t) & =m^{-1}\left(f^{-1} \circ \varphi(m(t)) \circ \varphi(m(s)) \circ f\right) \\
& =m^{-1}\left(f^{-1} \circ \varphi(m(t)) \circ f \circ f^{-1} \circ \varphi(m(s)) \circ f\right) \\
& =\varphi_{0}(s) \cdot \varphi_{0}(t) .
\end{aligned}
$$

Thus $\varphi_{0}$ is a homomorphism of the field structure of $R$ and, since $R$ is a real-closed field $\left[4\right.$, p. 273], $\varphi_{0}$ must be order preserving. Since $\varphi_{0}$ is identity on the rationals, 
$\varphi_{0}$ must then be the identity on $R$. In any event, $\varphi_{0}$ is a homeomorphism. Since $m$, $f_{0}$ and $m^{-1}$ are all homeomorphisms already, this forces $\varphi_{R, R}$ to be a homeomorphism. Now let $\pi: R \rightarrow T=R / Z$ be the natural projection. Every morphism $\beta \in \operatorname{Hom}(R, T)$ factors through $\pi$; that is, there is a unique morphism $\beta_{0} \in$ $\operatorname{Hom}(R, R)$ such that $\beta=\pi \circ \beta_{0}$. This map $\beta \mapsto \beta_{0}$ is continuous. Also the map Hom $(\varphi(R), \varphi(R)) \rightarrow \operatorname{Hom}(\varphi(T), \varphi(R))$ given by $\gamma \mapsto \gamma \circ \varphi_{R, T}(\pi)$ is clearly continuous. But we can write $\varphi_{R, T}$ as the composite of these last two maps and $\varphi_{R, R}$ :

$$
\varphi_{R, T}(\beta)=\varphi_{R, R}\left(\beta_{0}\right) \circ \varphi_{R, T}(\Pi) .
$$

We conclude that $\varphi_{R, T}$ is continuous, and so $T_{R}^{s}$ is an isomorphism.

We now apply the Five Lemma of homological algebra to the commutative diagram

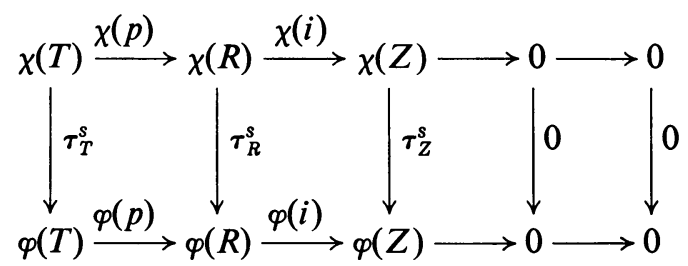

and conclude that $\tau_{Z}^{s}$ is at least a group isomorphism. Let $U$ be an open neighborhood of 0 in $\varphi(Z)$. Then

$$
\left(\tau_{Z}^{s}\right)^{-1}(U)=\chi(i)\left(\tau_{R}^{s}\right)^{-1}(\varphi(i))^{-1}(U) .
$$

Therefore $\tau_{Z}^{s}$ is continuous and is an isomorphism in $\mathscr{L}$ since $\chi(Z)$ is compact.

Next let $F_{n}$ be a finite cyclic group of order $n$. Then we have a short proper exact sequence

$$
0 \longrightarrow Z \stackrel{n \cdot 1_{z}}{\longrightarrow} Z \stackrel{p_{n}}{\longrightarrow} F_{n} \longrightarrow 0
$$

This brings us to the commutative diagram

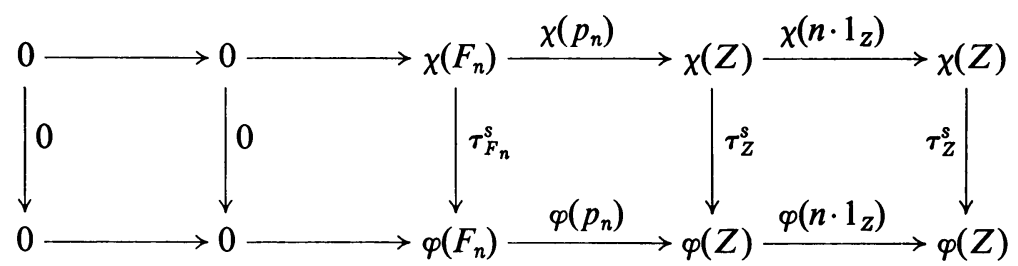

By the exactness of the rows and the Five Lemma, $\tau_{F}^{s}$ is an isomorphism.

We have shown that $\tau_{Z}^{s}, \tau_{R}^{s}, \tau_{T}^{s}$ and $\tau_{F_{n}}^{s}$, where $F_{n}$ is a finite cyclic group, are all isomorphisms in $\mathscr{L}$. We are going to show that $\tau^{s}$ is a natural equivalence, building up from these simple groups to more complicated groups. 
For $G$ and $H$ in $\mathscr{L}$, examine the following commutative diagram, which comes from applying $\chi$ and $\varphi$ to the direct sum diagram for $G \oplus H$ :

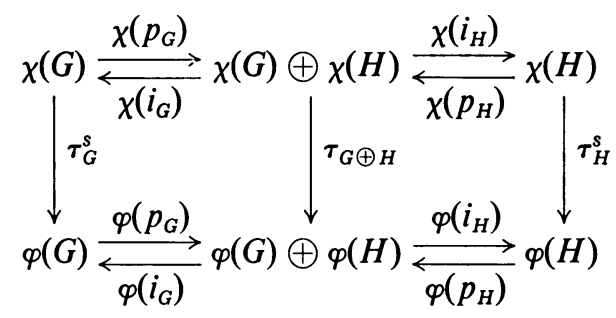

From this we see that

$$
\begin{aligned}
\tau_{G \oplus H}^{s} & =\tau_{G \oplus H} \circ \chi\left(p_{G}\right) \circ \chi\left(i_{G}\right)+\tau_{G \oplus H} \circ \chi\left(p_{H}\right) \circ \chi\left(i_{H}\right) \\
& =\varphi\left(p_{G}\right) \circ \tau_{G}^{s} \circ \chi\left(i_{G}\right)+\varphi\left(p_{H}\right) \circ \tau_{H}^{s} \circ \chi\left(i_{H}\right)=\tau_{G}^{s} \oplus \tau_{H}^{s} .
\end{aligned}
$$

Thus, if $\tau_{G}^{s}$ and $\tau_{H}^{s}$ are topological isomorphisms, then $\tau_{G \oplus H}^{s}$ is a topological isomorphism between $\chi(G \oplus H)$ and $\varphi(G \oplus H)$.

Let $G$ be a compact group in $\mathscr{L}$ and let $G$ be represented as a projective limit of $\left(\left\{G_{\alpha}\right\},\left\{f_{\alpha \beta}\right\}\right)$, where the $G_{\alpha}$ are all of the form $T^{n} \oplus F$, where $F$ is some finite group in $\mathscr{L}$. Then, since $F$ is the direct sum of finite cyclic groups, each $\tau_{G_{\alpha}}^{s}$ is an isomorphism in $\mathscr{L}$. We then obtain the following commutative diagram whenever $\alpha \leqq \beta$ :

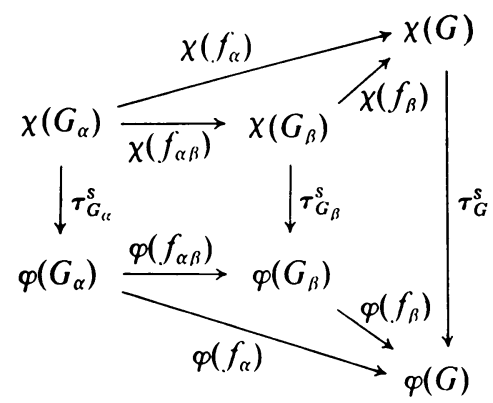

$\tau_{G}^{s}$ is a morphism in $\mathscr{L}$ because it is a homomorphism and $\chi(G)$ is discrete. The other arrows in the diagram all represent morphisms. $\varphi(G)$ is an inductive limit of the $\varphi\left(G_{\alpha}\right)$ and $\chi(G)$ is also an inductive limit of the $\varphi\left(G_{\alpha}\right)$ because it is an inductive limit of the $\chi\left(G_{\alpha}\right)$ and the $\tau_{G_{\alpha}}^{s}$ are all isomorphisms. Then by the uniqueness of morphisms in the definition of inductive limit in $\mathscr{L}, \tau_{G}^{s}$ must be an isomorphism.

Next let $G$ be a discrete group in $\mathscr{L}$. We refer the reader to the last part of the proof of Theorem 2. Let $\mu=\left(\tau_{Z}^{s}\right)^{-1}: \varphi(Z) \rightarrow \chi(Z)$, and let $\nu$ : Hom $(\varphi(G), \varphi(Z))$ $\rightarrow$ Hom $(\varphi(G), T)$ be the continuous homomorphism defined by $\nu(f)=\xi_{T} \circ \mu \circ f$ as in that proof. Because $\operatorname{Hom}(Z, G)$ is discrete, the function $\varphi_{Z, G}$ is a continuous homomorphism. Hence the function $\lambda=\nu \circ \varphi_{Z, G} \circ \xi_{G}^{-1}$ from $G$ to $\chi \varphi(G)$ is a morphism in $\mathscr{L}$. Hence $\delta=\chi(\lambda) \circ \rho_{\varphi(G)}: \varphi(G) \rightarrow \chi(G)$ is also a morphism in $\mathscr{L}$. From the rest of the proof we see that for any discrete object $G$, the morphism $\delta$ coincides 
with $\sigma_{G}^{\mu}$ as defined in condition (3) of Theorem 2, and that for any two discrete groups $G$ and $H$ in $\mathscr{L}$ and $f \in \operatorname{Hom}(G, H)$, the following diagram is commutative:

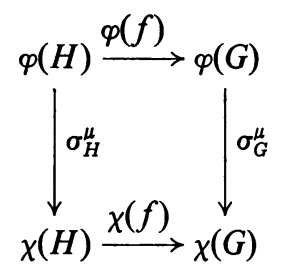

We now show that $\sigma_{G}^{\mu} \circ \tau_{G}^{s}=1_{\chi(G)}$ when $G$ is discrete: Let $\alpha \in \chi(G)$ and $y \in G$. Then

$$
\begin{aligned}
{\left[\left(\sigma_{G}^{\mu} \circ \tau_{G}^{s}\right)(\alpha)\right](y) } & =\left[\xi_{T} \circ \mu \circ \varphi_{Z, G}\left(\xi_{G}^{-1}(y)\right)\right]\left[\varphi_{G, T}(\alpha)(s)\right] \\
& =\left[\xi_{T} \circ \mu \circ \varphi_{Z, T}\left(\alpha \circ \xi_{G}^{-1}(y)\right)\right](s) \\
& =\left[\xi_{T} \circ\left(\tau_{Z}^{s}\right)^{-1} \circ\left(\tau_{Z}^{s}\right)\right]\left[\xi_{T}^{-1}(\alpha(y))\right]=\alpha(y) .
\end{aligned}
$$

In particular, if $G$ is a finitely generated discrete group in $\mathscr{L}$ then $G$ is a direct sum of cyclic groups and $\tau_{G}^{s}$ is an isomorphism; then $\sigma_{G}^{\mu}$ is also an isomorphism in $\mathscr{L}$. Finally, if $G$ is allowed to be any discrete group in $\mathscr{L}$, then we can write $\left(G,\left\{f_{\alpha}\right\}\right)$ as an inductive limit of $\left(\left\{G_{\alpha}\right\},\left\{f_{\alpha \beta}\right\}\right)$, where each $G_{\alpha}$ is a finitely generated group in $\mathscr{L}$. We are again led to a large commutative diagram whenever $\alpha \leqq \beta$ :

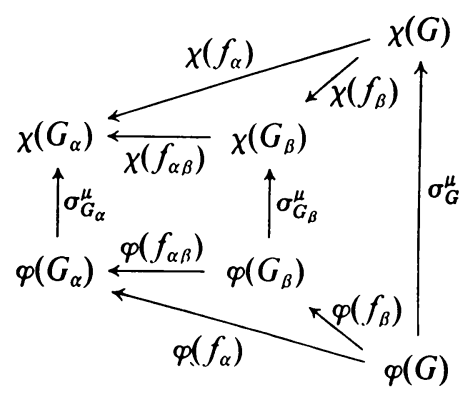

in which all the arrows are known to represent morphisms and $\sigma_{G_{\alpha}}^{\mu}$ and $\sigma_{G_{\beta}}^{\mu}$ are isomorphisms. Therefore both $\chi(G)$ and $\varphi(G)$ are projective limits of $\left(\left\{\varphi\left(G_{\alpha}\right)\right\}\right.$, $\left.\left\{\varphi\left(f_{\alpha \beta}\right)\right\}\right)$ and $\sigma_{G}^{\mu}$ must be an isomorphism. Therefore $\tau_{G}^{s}$ is also an isomorphism in $\mathscr{L}$.

Now let $G$ be an arbitrary object in $\mathscr{L}$. We can find a compact subgroup $G_{0}$ so that $G / G_{0}$ is without small subgroups. By what we have shown above and by the structure of groups in $\mathscr{L}$ without small subgroups, we know that $\tau_{G / G_{0}}^{s}$ is an isomorphism in $\mathscr{L}$. Also $\tau_{G_{0}}^{s}$ is. Applying $\chi$ and $\varphi$ to the exact sequence

$$
0 \longrightarrow G_{0} \stackrel{j}{\longrightarrow} G \stackrel{q}{\longrightarrow} G / G_{0} \longrightarrow 0
$$


gives us the following commutative diagram:

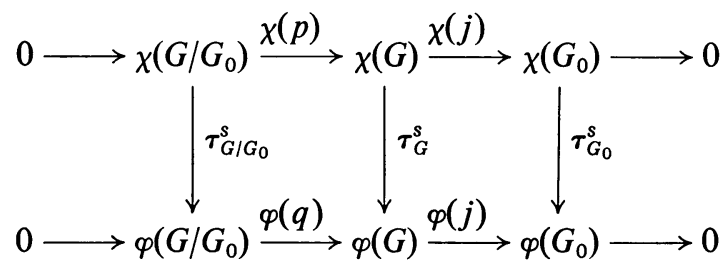

By the Five Lemma, $\tau_{G}^{s}$ is at least a group isomorphism. But $\chi\left(G_{0}\right)$ and $\varphi\left(G_{0}\right)$ are discrete groups. Hence the restriction of $\tau_{G_{0}}^{s}$ to the image of $\chi(q)$ is a topological isomorphism from an open subgroup of $\chi(G)$ to an open subgroup of $\varphi(G)$. From this we conclude that $\tau_{G}^{s}$ is an isomorphism in $\mathscr{L}$. Q.E.D.

THEOREM 4. Any contravariant functor $\varphi: \mathscr{L} \rightarrow \mathscr{L}$ which satisfies Axiom System II is naturally equivalent to the functor $\chi$.

Proof. This is basically a repetition of the proof of Theorem 3, with the following exception at the very end: after showing that $\tau_{G}^{s}$ is an isomorphism in $\mathscr{L}$ for groups without small subgroups, we express an arbitrary object $G$ of $\mathscr{L}$ as a proper projective limit $\left(G,\left\{f_{\alpha}\right\}\right)$ of an inverse system $\left(\left\{G_{\alpha}\right\},\left\{f_{\alpha \beta}\right\}\right)$ in $\mathscr{L}$ where each $G_{\alpha}$ is without small subgroups. We then have the following commutative diagram whenever $\alpha \leqq \beta$ :

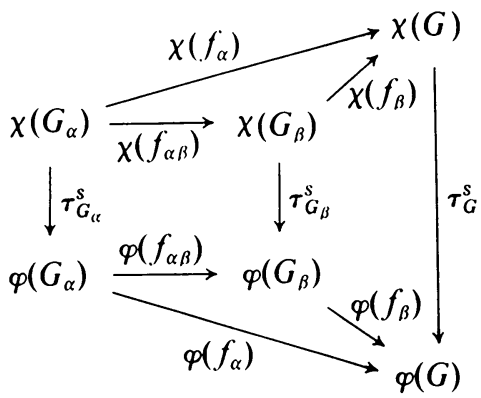

Now, the restriction of $\tau_{G}^{s}$ to the image of $\chi\left(f_{\alpha}\right)$ is a continuous homomorphism on an open subgroup of $\chi(G)$; therefore $\tau_{G}^{s}$ is a morphism in $\mathscr{L}$. But both $\left(\varphi(G),\left\{\varphi\left(f_{\alpha \beta}\right)\right\}\right)$ and $\left(\chi(G),\left\{\chi\left(f_{\alpha}\right) \circ\left(\tau_{G_{\alpha}}^{s}\right)^{-1}\right\}\right)$ are inductive limits of $\left(\left\{\varphi\left(G_{\alpha}\right)\right\},\left\{\varphi\left(f_{\alpha \beta}\right)\right\}\right)$ and so $\tau_{G}^{s}$ must be an isomorphism in $\mathscr{L}$. Q.E.D.

\section{4. $\chi$ is the only contravariant category equivalence in $\mathscr{L}$.}

Lemma 3. Let $\varphi: \mathscr{L} \rightarrow \mathscr{L}$ be a contravariant category equivalence. Then $\varphi$ takes inductive limits to projective limits and projective limits to inductive limits.

Proof. This is purely a categorical statement. Let $\left(G,\left\{f_{\alpha}\right\}\right)$ be an inductive limit of the direct system $\left(\left\{G_{\alpha}\right\},\left\{f_{\alpha \beta}\right\}\right)$. Then $\varphi$ induces morphisms $\varphi\left(f_{\alpha}\right): \varphi(G) \rightarrow \varphi\left(G_{\alpha}\right)$, where $\left(\left\{\varphi\left(G_{\alpha}\right)\right\},\left\{\varphi\left(f_{\alpha \beta}\right)\right\}\right)$ is an inverse system in $\mathscr{L}$, satisfying $\varphi\left(f_{\alpha \beta}\right) \circ \varphi\left(f_{\beta}\right)=\varphi\left(f_{\alpha}\right)$ 
whenever $\alpha \leqq \beta$. Let $H$ be an object in $\mathscr{L}$ and suppose $h_{\alpha}: H \rightarrow \varphi\left(G_{\alpha}\right)$ are morphisms such that $\varphi\left(f_{\alpha \beta}\right) \circ h_{\beta}=h_{\alpha}$ whenever $\alpha \leqq \beta$. We apply $\psi$ (an inverse for $\varphi$ ) and we see that $\left(\psi \varphi(G),\left\{\psi \varphi\left(f_{\alpha}\right)\right\}\right)$ is an inductive limit of the direct system $\left(\left\{\psi \varphi\left(G_{\alpha}\right)\right\},\left\{\psi \varphi\left(f_{\alpha \beta}\right)\right\}\right)$. Therefore there is a unique morphism $g: \psi \varphi(G) \rightarrow \psi(H)$ such that $g \circ \psi \varphi\left(f_{\alpha}\right)=\psi\left(h_{\alpha}\right)$ for every $\alpha$. Then, since $\psi_{H, \varphi(G)}$ is bijective, there is a morphism $g_{0}: H \rightarrow \varphi(G)$ such that $\psi\left(g_{0}\right)=g$, and $g_{0}$ is the unique morphism in $\operatorname{Hom}(H, \varphi(G))$ such that $\varphi\left(f_{\alpha}\right) \circ g_{0}$ $=h_{\alpha}$ for every $\alpha$. Thus $\left(\varphi(G),\left\{\varphi\left(f_{\alpha}\right)\right\}\right)$ is a projective limit of the inverse system $\left(\left\{\varphi\left(G_{\alpha}\right)\right\},\left\{\varphi\left(f_{\alpha \beta}\right)\right\}\right)$. The other half of the proof comes from reversing the arrows and the order of composition of morphisms in the above argument. Q.E.D.

Lemma 4. Let $\varphi: \mathscr{L} \rightarrow \mathscr{L}$ be a category equivalence. Let $G$ and $H$ be objects in $\mathscr{L}$ and let $f \in \operatorname{Hom}(G, H)$. Then

(1) $G \simeq\{0\}$ if and only if $\varphi(G) \simeq\{0\}$.

(2) $f=0$ if and only if $\varphi_{G, H}(f)=0$.

(3) $f$ is an isomorphism in $\mathscr{L}$ if and only if $\varphi(f)$ is an isomorphism in $\mathscr{L}$.

Proof. Recall that $\varphi$ is bijective on morphisms. Let $0_{G}: G \rightarrow G$ be the zero homomorphism. Then clearly $G \simeq\{0\}$ if and only if $0_{G}=1_{G}$. This proves (1).

Suppose $f=0$. Then we have maps $\alpha: G \rightarrow\{0\}$ and $\beta:\{0\} \rightarrow H$ such that $\beta \circ \alpha=f$. Therefore $\varphi(f)=\varphi(\alpha) \circ \varphi(\beta)$ factors through $\varphi(\{0\})$ and so $\varphi(f)=0$. This proves (2).

If $f$ is an isomorphism then $\varphi(f)$ is always an isomorphism for any functor $\varphi$. This proves (3). Q.E.D.

Lemma 5. Let $\varphi: \mathscr{L} \rightarrow \mathscr{L}$ be a category equivalence. Then $\varphi$ takes any proper morphism to a proper morphism and $\varphi$ is a proper exact functor.

Proof. In the category $\mathscr{L}$, every morphism has a kernel and cokernel. To see this, let $f: G \rightarrow H$ be a morphism in $\mathscr{L}$ and let $K=f^{-1}(0)$ be the ordinary grouptheoretic kernel of $f$, a closed subgroup of $G$. Then the natural inclusion $i: K \rightarrow G$ is a kernel of $f$ in the categorical sense; that is, $f \circ i=0$ and whenever $g: A \rightarrow G$ is a morphism in $\mathscr{L}$ such that $f \circ g=0$, then there exists a unique morphism $h: A \rightarrow K$ such that $g=i \circ h$. A kernel is unique up to canonical isomorphism in $\mathscr{L}$, so we may say that the kernel of any morphism in $\mathscr{L}$ is automatically a proper injection. Similarly, letting $H_{0}$ be the topological closure of the set-theoretical image of $f$ in $H$, we find that the natural proper surjection $p: H \rightarrow H / H_{0}$ is the cokernel of $f$, since the definition of cokernel merely reverses the arrows in the definition of kernel.

Now (we take the case when $\varphi$ is contravariant) because $\varphi$ is a category equivalence, we conclude from this arrow reversing process that $\varphi$ takes kernels to cokernels and vice versa. Suppose that $f: G \rightarrow H$ is a proper morphism and let $i$ and $p$ be the kernel and cokernel as above. Consider the natural map $q: G \rightarrow G / K$ which is clearly the cokernel of $i$. Then there is a unique morphism $f_{1}: G / K \rightarrow H$ such that $f=f_{1} \circ q . f_{1}$ is proper since $f$ is proper and $f_{1}$ is injective by construction. 
In fact, we see that $f_{1}$ is a kernel of $p$. Thus $f=f_{1} \circ q$ is the composition of a kernel and a cokernel in $\mathscr{L}$. Hence $\varphi(f)=\varphi(q) \circ \varphi\left(f_{1}\right)$ is also the composition of a kernel and a cokernel, both proper morphisms, so $\varphi(f)$ is proper.

Also, we can easily see that a short sequence

$$
0 \longrightarrow K \stackrel{f}{\longrightarrow} G \stackrel{g}{\longrightarrow} L \longrightarrow 0
$$

is proper exact if and only if $f$ is the kernel of $g$ and $g$ is the cokernel of $f$. Hence if such a sequence is proper exact, then in the induced sequence

$$
0 \longrightarrow \varphi(L) \stackrel{\varphi(g)}{\longrightarrow} \varphi(G) \stackrel{\varphi(f)}{\longrightarrow} \varphi(K) \longrightarrow 0,
$$

$\varphi(g)$ is the kernel of $\varphi(f)$ and $\varphi(f)$ is the cokernel of $\varphi(g)$ and so the induced sequence is proper exact. Q.E.D.

Lemma 6. Let $\varphi: \mathscr{L} \rightarrow \mathscr{L}$ be a contravariant category equivalence. Then $\varphi(Z) \simeq T$ and $\varphi(T) \simeq Z$.

Proof. Let $\psi: \mathscr{L} \rightarrow \mathscr{L}$ be an inverse for $\varphi$. Then we know that $\varphi \psi(T) \simeq T$. Since the map $\varphi_{Z, \psi(T)}: \operatorname{Hom}(Z, \psi(T)) \rightarrow \operatorname{Hom}(\varphi \psi(T), \varphi(Z))$ is bijective, there must be some nonzero morphism $\alpha$ from $\varphi \psi(T)$ to $\varphi(Z)$. Then $\varphi(Z)$ has a subgroup $K$ (namely the image of $\alpha$ ) which is isomorphic to $T$. Now the identity map $K \rightarrow K$ extends to a continuous homomorphism $\varphi(Z) \rightarrow K$ by the fact that any continuous character in $\mathscr{L}$ can be extended. This extended morphism $\varphi(Z) \rightarrow K$ thus splits and hence $K$ occurs as a direct factor in $\varphi(Z)$; that is, $\varphi(Z) \simeq T \oplus H$ for some object $H$ in $\mathscr{L}$. But then by Lemmas 1 and 5 ,

$$
Z \simeq \psi \varphi(Z) \simeq \psi(T) \oplus \psi(H),
$$

which is impossible unless $\psi(H)$ is $\{0\}$, forcing $H \simeq\{0\}$ and $\varphi(Z) \simeq T$. Also then $\psi(T) \simeq Z$. By applying the same argument first to $\psi$, we can show that $\psi(Z) \simeq T$ and $\varphi(T) \simeq Z$. Q.E.D.

THEOREM 5. Any contravariant category equivalence $\varphi: \mathscr{L} \rightarrow \mathscr{L}$ is naturally equivalent to the functor $\chi$.

Proof. We show that $\varphi$ satisfies Axiom System I and then apply Theorem 3 . Lemmas 3 and 5 show that $\varphi$ satisfies all the axioms of Axiom System I except possibly $\mathrm{Ia}(\varphi(R) \simeq R)$. By Lemma 6 we have $\varphi(Z) \simeq T$ and $\varphi(T) \simeq Z$. We can also show $\varphi(R) \simeq R$, as follows. The short proper exact sequence

$$
0 \longrightarrow Z \stackrel{i}{\longrightarrow} R \stackrel{p}{\longrightarrow} T \longrightarrow 0
$$

is taken by $\varphi$ into a short proper exact sequence

$$
0 \longrightarrow Z \stackrel{\varphi(p)}{\longrightarrow} \varphi(R) \stackrel{\varphi(i)}{\longrightarrow} T \longrightarrow 0 .
$$


We can find a compact set $C$ in $\varphi(R)$ whose image under $\varphi(i)$ is all of $T$. Letting $x_{0}$ be the image of 1 under $\varphi(p)$, we see that $C \cup\left\{x_{0}\right\}$ generates $\varphi(R)$; hence $\varphi(R)$ is compactly generated. Also, since the kernel of $\varphi(i)$ is discrete, $\varphi(R)$ and $\varphi(T)$ are locally isomorphic. Therefore $\varphi(R)$ is a group without small subgroups, because $T$ is. These facts taken together mean that $\varphi(R)$ is isomorphic to an object in $\mathscr{L}$ of the form

$$
R^{j} \oplus T^{m} \oplus Z^{n} \oplus F,
$$

where $F$ is a direct sum of finite cyclic groups. Each of these finite cyclic groups is isomorphic with a subgroup of $T$ and a quotient group of $Z$; hence $\varphi$ takes each one to a quotient group of $Z$ and a subgroup of $T$; i.e., a finite cyclic group. Let $\psi$ be an inverse of $\varphi$; then all these statements hold for $\psi$ as well. Thus $\varphi \psi(R)$ is of the form

$$
\varphi(R)^{j} \oplus Z^{m} \oplus T^{n} \oplus F_{1},
$$

where $F_{1}$ is a finite group. But $\varphi \psi(R) \simeq R$ and $R$ cannot be expressed as a nontrivial direct sum of objects in $\mathscr{L}$. Therefore one of the factors in this representation must be isomorphic with $R$ and the others must all be trivial. The only possibility for this is $j=1, \varphi(R) \simeq R, m=0, n=0$ and $F_{1} \simeq\{0\}$. Q.E.D.

5. Some variations. The following result is weaker than Theorem 5 , but its proof is much shorter because it does not depend on Theorem 3.

TheOREM 6. Let $\varphi: \mathscr{L} \rightarrow \mathscr{L}$ be a contravariant category equivalence which is also a topological functor in $\mathscr{L}$. Then $\varphi$ is naturally equivalent to $\chi$.

Proof. By Lemmas 1, 2 and 5, $\varphi$ is additive; by Lemma 6, $\varphi(T) \simeq Z$. Let $s$ be a generator of $\varphi(T)$. Consider the natural transformation $\tau^{s}: \chi \rightarrow \varphi$ discussed in Theorem 1. Since $\varphi_{G, T}$ is bijective, each $\tau_{G}^{s}$ is a bijective morphism in $\mathscr{L}$. In particular, then, $\tau_{Z}^{s}: \chi(Z) \rightarrow \varphi(Z)$ is an isomorphism in $\mathscr{L}$ because $\chi(Z)$ is compact. Let $\mu=\left(\tau_{Z}^{s}\right)^{-1}$ and define, for each $G$ in $\mathscr{L}$, a morphism $\theta_{G}: \chi(G) \rightarrow \chi(G)$ by $\theta_{G}=\sigma_{G}^{\mu} \circ \tau_{G}^{s}$, where $\sigma^{\mu}$ is the natural transformation defined in Theorem 2. Then $\theta=\left\{\theta_{G} ; G\right.$ in $\left.\mathscr{L}\right\}$ is a natural transformation from $\chi$ to $\chi$. Furthermore,

$$
\theta_{Z}=\sigma_{Z}^{\mu} \circ \tau_{Z}^{s}=1_{\chi(Z)} \text {. }
$$

But then by Theorem 2, $\theta$ must coincide with the natural transformation $\eta: \chi \rightarrow \chi$ defined by $\eta_{G}=1_{\chi(G)}$ for every object $G$. Hence each $\tau_{G}^{s}$ is a topological isomorphism. Q.E.D.

The following alternate proof of Theorem 4 involves a different approach which was suggested by the referee. It utilizes the concepts of category with convergence and continuous functor; see [3] for further explication and applications of these ideas, especially the examples in paragraph 2.2, p. 117 .

THEOREM 4. Any contravariant functor $\varphi: \mathscr{L} \rightarrow \mathscr{L}$ which satisfies Axiom System II is naturally equivalent to the functor $\chi$. 
Proof. Let $\mathscr{K}$ be the category of all proper inverse systems in $\mathscr{L}$. Then $(\mathscr{L}, \mathscr{K})$ is a category with convergence. Let $\mathscr{L}_{1}$ be the full subcategory of $\mathscr{L}$ whose objects are the groups without small subgroups; then $\left(\mathscr{L}_{1}, \mathscr{K} \mid \mathscr{L}_{1}\right)$ is the subcategory with convergence induced from $\mathscr{K}$, and $\mathscr{L}_{1}$ is dense in $(\mathscr{L}, \mathscr{K})$ because every group in $\mathscr{L}$ is a proper projective limit of groups in $\mathscr{L}_{1}$. Now let $\mathscr{L}_{2}$ be the full subcategory of $\mathscr{L}_{1}$ whose objects are all groups in $\mathscr{L}$ isomorphic to one of the form $R^{j} \oplus Z^{m}$ $\oplus T^{n} \oplus F$, where $F$ is a finite abelian group. These are the compactly generated groups without small subgroups in $\mathscr{L}$, or the elementary groups. Let $\left(\mathscr{L}_{3}, \mathscr{K}_{3}\right)$ be the category with coconvergence where $\mathscr{L}_{3}$ is the subcategory of $\mathscr{L}_{1}$ whose objects are all discrete groups in $\mathscr{L}$, and $\mathscr{K}_{3}$ is the category of all proper direct systems in $\mathscr{L}_{3}$. Then $\left(\mathscr{L}_{4}, \mathscr{K}_{3} \mid \mathscr{L}_{4}\right)$ is the full subcategory (with coconvergence) of $\mathscr{L}_{3}$ and of $\mathscr{L}_{2}$ whose objects are the finitely generated discrete groups; $\mathscr{L}_{4}$ is codense in $\mathscr{L}_{3}$. Finally, $\mathscr{L}_{5}$ is the skeletal subcategory of $\mathscr{L}_{2}$ whose objects are the groups $R^{j} \oplus Z^{m}$ $\oplus T^{n} \oplus F$, where $F$ is a finite direct sum of cyclic quotient groups $Z_{p^{n}}$ of $Z$ of prime power order. Now, since $\varphi(R) \simeq R$ by Axiom IIa, we can steal the first part of the proof of Theorem 3 and show that $\varphi(T) \simeq Z$. Choosing a generator $s$ of $\varphi(T)$, we let $\tau^{s}: \chi \rightarrow \varphi$ be the pseudo-natural transformation guaranteed by Theorem 1. (Actually, we need only define $\tau_{G}^{s}$ for $G \in \mathscr{L}_{5}$.) Again following the proof of Theorem 3, we arrive at the fact that $\tau^{s}$ is a natural equivalence between the restricted functors $\chi \mid \mathscr{L}_{5}$ and $\varphi \mid \mathscr{L}_{5}$, since $\tau_{R}^{s}, \tau_{T}^{s}, \tau_{Z}^{s}$ and $\tau_{Z_{n}}^{s}$ are isomorphisms, and $\tau_{G \oplus H}^{s}=\tau_{G}^{s} \oplus \tau_{H}^{s}$. The reasoning from this point is as follows: Since $\mathscr{L}_{5}$ is a skeleton of $\mathscr{L}_{2}, \tau^{s}$ extends uniquely to a natural equivalence $\tau^{s}: \chi\left|\mathscr{L}_{2} \rightarrow \varphi\right| \mathscr{L}_{2}$. (This extension must agree with $\tau^{s}$ by the proof of Theorem 1.) By restricting, we see that $\chi\left|\mathscr{L}_{4} \simeq \varphi\right| \mathscr{L}_{4}$. Then (Axiom IIc), since $\chi$ and $\varphi$ are cocontinuous on $\left(\mathscr{L}_{3}, \mathscr{K}_{3}\right)$, this extends by the codensity of $\mathscr{L}_{4}$ in $\mathscr{L}_{3}$ to the natural equivalence $\tau^{s}: \chi\left|\mathscr{L}_{3} \rightarrow \varphi\right| \mathscr{L}_{3}$. Since every object in $\mathscr{L}_{1}$ is isomorphic to a direct sum of groups in $\mathscr{L}_{2}$ and $\mathscr{L}_{3}$, we conclude that $\tau^{s}: \chi\left|\mathscr{L}_{1} \rightarrow \varphi\right| \mathscr{L}_{1}$ is a natural equivalence. Since we know that $\chi$ and $\varphi$ are continuous on $\mathscr{L}$ and $\mathscr{L}_{1}$ is dense in $\mathscr{L}$, we conclude that $\tau^{s}$ extends to a natural equivalence between $\chi$ and $\varphi$.

6. Pseudo-natural transformations between covariant functors. The following two theorems are analogous to Theorems 1 and 2.

THEOREM 7. Let $\varphi: \mathscr{L} \rightarrow \mathscr{L}$ be a covariant functor. Let $s$ be any element of $\varphi(Z)$. Then there is exactly one pseudo-natural transformation $\eta^{s}: \iota \rightarrow \varphi$ satisfying the equation $\eta_{Z}^{s}(1)=s$. Each $\eta_{G}^{s}: G \rightarrow \varphi(G)$ is defined by the formula

$$
\eta_{G}^{s}(x)=\left[\varphi_{Z, G}\left(\xi_{G}^{-1}(x)\right)\right](s) \text { for } x \in G .
$$

Thus there is a bijective correspondence between pseudo-natural transformations $\eta^{s}: \iota \rightarrow \varphi$ and elements $s$ of $\varphi(Z)$. If $\varphi$ is a topological additive functor, then each $\eta^{s}$ is in fact a natural transformation (that is, each $\eta_{G}^{s}$ is a morphism in $\mathscr{L}$ ).

Proof. Let $s \in \varphi(Z)$ and let $\eta^{s}$ be defined as above. Let $G$ and $H$ be objects of $\mathscr{L}$ 
and let $f \in \operatorname{Hom}(G, H)$. Then for $x \in G$, we have

while

$$
\begin{aligned}
\left(\varphi(f) \circ \eta_{G}^{s}\right)(x) & =\varphi_{G, H}(f)\left(\left[\varphi_{Z, G}\left(\xi_{G}^{-1}(x)\right)\right](s)\right) \\
& =\left[\varphi_{Z, H}\left(f \circ \xi_{G}^{-1}(x)\right)\right](s),
\end{aligned}
$$

$$
\left(\eta_{H}^{s} \circ f\right)(x)=\left[\varphi_{Z, H}\left(\xi_{H}^{-1}[f(x)]\right)\right](s) .
$$

But we saw in the proof of Theorem 2 that $f \circ \xi_{G}^{-1}(x)=\xi_{H}^{-1}(f(x))$. Thus $\eta^{s}=\left\{\eta_{G}^{s}\right\}$ is a pseudo-natural transformation. Furthermore,

$$
\eta_{Z}^{s}(1)=\left[\varphi_{Z, Z}\left(\xi_{Z}^{-1}(1)\right)\right](s)=\left[\varphi_{Z, Z}\left(1_{Z}\right)\right](s)=1_{\varphi(Z)}(s)=s .
$$

Now let $\eta: \iota \rightarrow \varphi$ be any pseudo-natural transformation, and let $s=\eta_{Z}(1)$. We show that $\eta=\eta^{s}$. Let $G$ be an object in $\mathscr{L}$ and let $x \in G$. Then since $\eta$ is a pseudonatural transformation, the following diagram is commutative for the morphism $\xi_{G}^{-1}(x) \in \operatorname{Hom}(Z, G)$ :

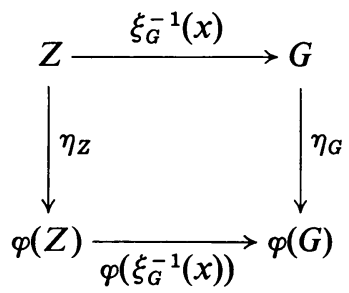

Using this information, we see that

$$
\begin{aligned}
\eta_{G}^{s}(x) & =\left[\varphi_{Z, G}\left(\xi_{G}^{-1}(x)\right)\right]\left[\eta_{Z}(1)\right] \\
& =\eta_{G}\left[\left(\xi_{G}^{-1}(x)\right)(1)\right]=\eta_{G}(x) .
\end{aligned}
$$

Finally, we see that the map $G \rightarrow \varphi(G)$ given by $x \mapsto \eta_{G}^{s}(x)$ is obtained by first applying the map $\xi_{G}^{-1}$ from $G$ to $\operatorname{Hom}(Z, G)$, then the map $\varphi_{Z, G}$ from $\operatorname{Hom}(Z, G)$ to Hom $(\varphi(Z), \varphi(G))$ and then the map Hom $(\varphi(Z), \varphi(G)) \rightarrow \varphi(G)$ given by $f \mapsto f(s)$. Therefore if $\varphi_{Z, G}$ is a continuous homomorphism, our map $\eta_{G}^{s}$ is a morphism in $\mathscr{L}$. This shows the last statement of Theorem 7. Q.E.D.

Incidentally, applying Theorem 7 to $\varphi=\chi^{2}$ tells us that the natural transformation $\rho: \iota \rightarrow \chi^{2}$ is determined uniquely even as a pseudo-natural transformation, once we specify the element $\rho_{Z}(1)$ in $\chi^{2}(Z)$. This fact is recorded more as a curiosity than a result.

THEOREM 8. Let $\varphi: \mathscr{L} \rightarrow \mathscr{L}$ be a convariant functor. Let $\mu: \varphi(T) \rightarrow T$ be a function (not necessarily a morphism in $\mathscr{L}$ ). Then the following two conditions are equivalent:

(1) There exists a pseudo-natural transformation $\omega^{\mu}: \varphi \rightarrow \iota$ such that $\omega_{T}^{\mu}=\mu$.

(2) For every object $G$ of $\mathscr{L}$ and every $x \in \varphi(G)$, the following function $\chi(G) \rightarrow T$ is a morphism in $\mathscr{L}$ :

$$
\alpha \mapsto\left[\mu \circ\left(\varphi_{G, T}(\alpha)\right)\right](x) .
$$

In any case, there is at most one pseudo-natural transformation satisfying (1). In 
fact, if $\omega^{\mu}$ satisfies (1), then for $G$ in $\mathscr{L}$ and $x \in \varphi(G), \omega_{G}^{\mu}(x)$ is that unique element of $G$ which satisfies

$$
\alpha\left(\omega_{G}^{\mu}(x)\right)=\left[\mu \circ\left(\varphi_{G, T}(\alpha)\right)\right](x) \text { for every } \alpha \in \chi(G) .
$$

In particular, if $\varphi$ is topological and additive and $\mu$ is a morphism in $\mathscr{L}$, then condition (2) is satisfied and the $\omega^{\mu}$ satisfying condition (1) is actually a natural transformation.

Proof. First, suppose (2) is satisfied. Then the existence and uniqueness of $\omega_{G}^{\mu}(x)$ in $G$ satisfying condition (3) is guaranteed by the Pontryagin Duality Theorem, and so we define $\omega^{\mu}$ by condition (3). We show that $\omega^{\mu}$ satisfies condition (1). Let $G$ and $H$ be objects in $\mathscr{L}$, let $f \in \operatorname{Hom}(G, H)$, let $x \in \varphi(G)$, and let $\alpha \in \chi(H)$. Then

$$
\begin{aligned}
\alpha\left[\left(\omega_{H}^{\mu} \circ \varphi(f)\right)(x)\right] & =\mu\left[\varphi_{H, T}(\alpha)\left(\varphi_{G, H}(f)(x)\right)\right] \\
& =\mu\left[\varphi_{G, T}(\alpha \circ f)(x)\right] \\
& =(\alpha \circ f)\left[\omega_{G}^{\mu}(x)\right] \\
& =\alpha\left[\left(f \circ \omega_{G}^{\mu}\right)(x)\right] .
\end{aligned}
$$

This shows that $\omega^{\mu}$ is a pseudo-natural transformation. Now let $x \in \varphi(T)$ and note that $1_{T} \in \chi(T)$. Then we have

$$
\omega_{T}^{\mu}(x)=1_{T}\left[\omega_{T}^{\mu}(x)\right]=\left[\mu \circ\left(\varphi_{T, T}\left(1_{T}\right)\right)\right](x)=\mu(x) .
$$

Thus $\omega^{\mu}$ satisfies condition (1).

Now suppose $\omega: \varphi \rightarrow \iota$ satisfies condition (1). Let $G$ be an object in $\mathscr{L}$, let $x \in \varphi(G)$ and let $\alpha \in \chi(G)$. Then, since $\omega$ is a pseudo-natural transformation, we have

$$
\alpha\left(\omega_{G}(x)\right)=\left(\alpha \circ \omega_{G}\right)(x)=\left(\omega_{T} \circ \varphi_{G, T}(\alpha)\right)(x)=\left[\mu \circ \varphi_{G, T}(\alpha)\right](x) .
$$

Hence the function $\chi(G) \rightarrow T$ in condition (2) is simply the map $\alpha \mapsto \alpha\left(\omega_{G}(x)\right.$ ), so condition (2) is satisfied, and we have shown that $\omega_{G}(x)=\omega_{G}^{\mu}(x)$, where $\omega^{\mu}$ is defined by condition (3). Thus $\omega=\omega^{\mu}$, showing the uniqueness of $\omega^{\mu}$.

Now let $\varphi$ be topological and additive and let $\mu$ be a morphism in $\mathscr{L}$. Then clearly condition (2) holds, and so $\omega^{\mu}$ exists satisfying condition (1). We demonstrate another way of constructing $\omega^{\mu}$. Let $G$ be an object of $\mathscr{L}$. Let $\lambda$ : Hom $(\varphi(G), \varphi(T))$ $\rightarrow$ Hom $(\varphi(G), T)$ be defined by $\lambda(f)=\mu \circ f$. As in the case of $\nu$ in the proof of Theorem $2, \lambda$ is a continuous homomorphism between topological groups. Now define $\beta=\lambda \circ \varphi_{G, T} . \beta$ is a morphism from $\chi(G)$ to $\chi \varphi(G)$. Finally define $\varepsilon=\rho_{G}^{-1} \circ \chi(\beta)$ - $\rho_{\varphi(G)}$, a morphism from $\varphi(G)$ to $G$. We claim that $\varepsilon=\omega_{G}^{\mu}$. To see this, let $x \in \varphi(G)$ and $\alpha \in \chi(G)$. Then

$$
\begin{aligned}
\alpha(\varepsilon(X)) & =\left[\left(\chi(\beta) \circ \rho_{\varphi(G)}\right)(x)\right](\alpha) \\
& =\left(\rho_{\varphi(G)}(x) \circ \beta\right)(\alpha) \\
& =\rho_{\varphi(G)}(x)\left[\left(\lambda \circ \varphi_{G, T}\right)(\alpha)\right] \\
& =\left[\mu \circ \varphi_{G, T}(\alpha)\right](x)=\alpha\left[\omega_{G}^{\mu}(x)\right] .
\end{aligned}
$$

Therefore $\omega^{\mu}$ is a natural transformation. Q.E.D. 


\section{REFERENCES}

1. I. Bicur and A. Deleanu, Introduction to the theory of categories and functors, Wiley, New York, 1968.

2. E. Hewitt and K. A. Ross, Abstract harmonic analysis. Vol. I: Structure of topological groups. Integration theory, group representations, Die Grundlehren der math. Wissenschaften, Band 115, Academic Press, New York and Springer-Verlag, Berlin, 1963. MR 28 \#158.

3. K. H. Hofmann, Categories with convergence, exponential functors, and the cohomology of compact abelian groups, Math. Z. 104 (1968), 106-140. MR 37 \#4195.

4. S. Lang, Algebra, Addison-Wesley, Reading, Mass., 1965. MR 33 \#5416.

5. S. Mac Lane, Homology, Die Grundlehren der math. Wissenschaften, Band 114, Academic Press, New York, and Springer-Verlag, Berlin, 1963. MR 28 \#122.

6. B. Mitchell, Theory of categories, Pure and Appl. Math., vol. 17, Academic Press, New York, 1965. MR 34 \#2647.

7. M. Moskowitz, Homological algebra in locally compact abelian groups, Trans. Amer. Math. Soc. 127 (1967), 361-404. MR 35 \#5861.

8. L. S. Pontryagin, Topological groups, GITTL, Moscow, 1954; English transl., Gordon and Breach, New York, 1966. MR 17, 171; MR 34 \#1439.

9. K. Suzuki, Notes on the duality theorem of non-commutative topological groups, Tôhoku Math. J. (2) 15 (1963), 182-186. MR 26 \#6293.

10. A. Weil, L'intégration dans les groupes topologiques et ses applications, Hermann, Paris, 1951.

UNIVERSITY OF CALIFORNIA,

Santa Barbara, California 93106

Dartmouth College,

HaNover, New Hampshire 03755 